\title{
The thick strip method for slender body fluid structure interaction
}

\author{
Spencer Sherwin, Mohsen Lahooti, Yan Bao, Mike Graham and Rafael Palacios \\ Department of Aeronautics \\ Imperial College London \\ South Kensington, London, UK \\ e-mail: s.sherwin@imperial.ac.uk, web page: http://www.imperial.ac.uk/people/s.sherwin
}

\begin{abstract}
For slender body fluid structure interaction which arise in problems such as the vortex induced vibration of oil riser pipes[1], flexible wings [2] or parked wind turbine blades, there is a natural separation of spatial scales between the fluid and structural problem. Nevertheless the large scale dynamics of the structure can have a notable impact on the fluid flow, for example leading to large scale separation which then modify the fluid forces applied to the structure. To resolve the full scale fluid structure interaction problem at realistic flow conditions/Reynolds number is typically prohibitive even on the largest HPC systems currently available.
\end{abstract}

A reasonable modelling approach to address these challenging problems is to leverage the scale separation between the fluid and structural problem and only model the fluid on a strip at a series of locations along the slender body. Earlier approaches to this type of modelling used potential flow, two-dimensional U-RANS or even empirical data, in "thin" strip approximations. However these approaches were unable to capture the near body anisotropic or transitional flow features which are often responsible for energising the slender body dynamics.

In [1] we proposed a generalized "thick" strip method where we adopt a finite thickness strip, which is still thin compared to the slender body, within which we apply an under-resolved Direct Numerical Simulation uDNS or implicit Large Eddy Simulation iLES modelling to capture the anisotropic flow behaviour and if sufficiently resolved the transitional nature of the flow. Within each strip we apply a high fidelity spectral/hp element-Fourier approximation using the Nektar++ package [3].

In this presentation we will outline the development and application of the thick strip modelling method for vortex induced problem of riser pipes and wind turbine blades. We will also discuss the challenges of the high fidelity modelling using spectral/hp element approximations to capture the unsteady anisotropic flow.

\section{REFERENCES}

[1] Y. Bao, R. Palacios, M. Graham, S.J. Sherwin, Generalized thick strip modelling for vortexinduced vibration of long flexible cylinders, J. Comp. Phys, v 321, 2016.

[2] M. Lahooti, R. Palacios, S.J. Sherwin, Thick Strip Method for Efficient Large-Eddy Simulation of Flexible Wings in Stall AIAA 2021-0363, 2021.

[2] www.nektar.info. 